\title{
A HIGH FREQUENCY ELECTROMAGNETIC IMPEDANCE IMAGING SYSTEM
}

\author{
Hung-Wen Tseng, Lawrence Berkeley National Laboratory, Berkeley, CA \\ Ki Ha Lee, Lawrence Berkeley National Laboratory, Berkeley, CA \\ Alex Becker, University of California, Berkeley, CA
}

\begin{abstract}
Non-invasive, high resolution geophysical mapping of the shallow subsurface is necessary for delineation of buried hazardous wastes, detecting unexploded ordinance, verifying and monitoring of containment or moisture contents, and other environmental applications. Electromagnetic (EM) techniques can be used for this purpose since electrical conductivity and dielectric permittivity are representative of the subsurface media. Measurements in the EM frequency band between 1 and 100 $\mathrm{MHz}$ are very important for such applications, because the induction number of many targets is small and the ability to determine the subsurface distribution of both electrical properties is required. Earlier workers were successful in developing systems for detecting anomalous areas, but quantitative interpretation of the data was difficult. Accurate measurements are necessary, but difficult to achieve for high-resolution imaging of the subsurface. We are developing a broadband non-invasive method for accurately mapping the electrical conductivity and dielectric permittivity of the shallow subsurface using an EM impedance approach similar to the MT exploration technique. Electric and magnetic sensors were tested to ensure that stray EM scattering is minimized and the quality of the data collected with the high-frequency impedance (HFI) system is good enough to allow high-resolution, multi-dimensional imaging of hidden targets. Additional efforts are being made to modify and further develop existing sensors and transmitters to improve the imaging capability and data acquisition efficiency.
\end{abstract}

\section{Introduction}

Accurate information on the shallow $(<10 \mathrm{~m})$ subsurface distribution in electrical conductivity and dielectric permittivity, which are related to local geochemistry and geohydrology, is useful in nearsurface engineering, and increasingly more important in environmental studies. At the low end of the electromagnetic $(\mathrm{EM})$ geophysical spectrum $(<300 \mathrm{kHz})$, DC resistivity and loop-loop induction measurements with small spacings have served adequately to map the near-surface resistivity variations since electrical conduction dominates in this low frequency band. However, the DC resistivity and induction methods suffer from lack of resolution and insensitivity to medium permittivity, which is primarily dependant on water content. Also, DC resistivity methods tend to be slow and expensive to deploy and the requirement of planting electrodes in the earth can be undesirably invasive in hazardous environments. Low-frequency inductive EM measurements are much more rapid but are frequently less informative, particularly in complex environments. At frequencies higher than $50 \mathrm{MHz}$, dielectric property (permittivity) starts dominating the EM response. For this reason ground-penetrating radar (GPR) is widely used to investigate shallow subsurface moisture content variations. Though GPR can provide excellent resolution with efficiency in field data acquisition, the method is often limited in its depth of investigation due to the strong attenuation of high-frequency EM waves in conductive media. Given the low amplitude induction response of many small near-surface targets and the need to determine both the electric conductivity and dielectric permittivity, use of frequencies between $100 \mathrm{kHz}$ 
and $100 \mathrm{MHz}$ for geophysical exploration is necessary. The term 'high frequency' as used herein refers to this region of the EM spectrum.

A baseline study (Pellerin et al., 1997) shows that electromagnetic instruments in the low frequency $(<300 \mathrm{kHz})$ and GPR systems $(>30 \mathrm{MHz})$ are well developed in the commercial sector. However, instrumentation suitable for collecting calibrated field data in the high frequency range is critically lacking. Stewart et al. (1994) produced a loop-loop-like system for measuring the ellipticity and tilt angle of the received magnetic field. However it appeared to have a limited range of application.

Using the frequency band between $100 \mathrm{kHz}$ and $100 \mathrm{MHz}$ and continuing the work of Frangos (2000) we are developing a broadband high-frequency impedance (HFI) system (Lee and Becker, 2001, Tseng et al., 2003) that measures the ratio of the electric and magnetic fields produced by a loop source. The HFI system offers the ability to determinine both the resistivity and permittivity at depths to a few meters. This is a surface-based and completely non-invasive technique, it is also allows rapid and inexpensive acquisition of field data. Song et al. (2002) give an analysis of a plane-wave HFI technique that operates in this spectral region. As a practical matter, generating plane waves at these frequencies is inconvenient because of the difficulty involved in building a sufficiently powerful transmitter required for large propagation distances while avoiding interference with other EM device users. Frangos (2000) completed a proof-of-concept form of the HFI system utilizing off-the-shelf instrumentation. It was tested in controlled field conditions. The incident EM wave was generated by a magnetic loop transmitter for near-field EM wave measurements. The system determines the EM impedance at selected frequencies between $100 \mathrm{kHz}$ and $30 \mathrm{MHz}$ from direct measurement of continuous wave electric and magnetic fields at source-to-detector separations between four and sixteen meters.

Encouraged with the success of the prototype, we are upgrading the system to reduce spurious scattering and increase signal level to improve its reliability and accuracy, and to extend the operating frequency to $100 \mathrm{MHz}$.

\section{Theoretical background and field measurements}

The electrical conductivity and dielectric permittivity determine the behavior of EM fields as they diffuse and travel through subsurface materials. The EM fields may be measured on or above the ground surface. For practical considerations, impedance, the ratio of the electric field to the corresponding orthogonal magnetic field, is used to infer the subsurface electrical properties. Unlike magnetic field ellipticity and tilt angles, the impedance is easy to use for data interpretation by inversion as no information is required about the source function.

For plane wave incidence, the surface impedance, $Z$, for a homogeneous isotropic medium at a fixed angular frequency, $\omega$, can be written as

$$
Z=\frac{E_{x}}{H_{y}}=\frac{\mu \omega}{\sqrt{\varepsilon \mu \omega^{2}-i \mu \sigma \omega}},
$$

where $E x$ and $H y$ are orthogonal electric and magnetic fields; $\varepsilon$ is the dielectric permittivity; $\mu$ is the magnetic permeability; $\sigma$ is the electric conductivity; and $i=\sqrt{-1}$ is the imaginary operator. The plane-wave impedance, as is shown, is useful in understanding the relationship between the impedance and electrical parameters. Apparently, the impedance is most affected by the dielectric permittivity at high frequencies and by the electrical conductivity at low frequencies. In practice, however, it is preferable to use the general EM impedance, $Z=E_{x} / H_{y}$, where fields are no longer plane wave. This is because power and signal strength considerations require relatively close separations between the 
transmitter and receiver. The general form of impedance over a layered earth can be easily calculated using the modeling program, EM1D.

To demonstrate the relationship between the general EM impedance and the electrical properties, a three-layer model as sketched in Figure 1(a) is considered. The model represents the near-surface electrical structure of a vineyard in northern California selected for monitoring water content in the soil. Due to soil types and various water content, the soil resistivity are set to 40, 10 and $40 \mathrm{ohm}-\mathrm{m}$ and the dielectric constants 16, 19, and 25 from the top to the bottom layers. The thickness of the top and the second layers are 0.5 and $1 \mathrm{~m}$, respectively. Using a magnetic loop dipole source placed $1 \mathrm{~m}$ above the ground surface and polarized in the horizontal $y$-direction, we calculated $E_{x}$ the electric field in the horizontal $x$-direction, and $H_{y}$ the magnetic field in the $y$-direction, at the same height as the source and $8 \mathrm{~m}$ away from it. We then obtained the EM impedance by taking the ratio, i.e. $E_{x} / H_{y}$. This setup is similar to the coplanar loop-loop EM method, but the electric field is also required at the observation location for the impedance estimates. The amplitude and phase of the impedance for this model over the frequency range from $100 \mathrm{kHz}$ and $100 \mathrm{MHz}$ are represented by the bold solid line in Figure 1(b) and 1(c), respectively. A perturbation of the dielectric constant of the top layer from 16 to 13 results in a different impedance as represented by the solid circles in the figure, while a change in resistivity of the top layer from 40 to $80 \mathrm{ohm}-\mathrm{m}$ (open circles) also reveals the sensitivity of the impedance values to subsurface electrical properties. While keeping both parameters in each layer intact but increasing the thickness of the top layer by $0.1 \mathrm{~m}$ to $0.6 \mathrm{~m}$, the impedance indicated by crosses in Figure 1(b) and 1(c) also shows a pronounced amplitude change at almost all frequencies.

\section{Instrumentation}

The HFI system described in Frangos (2000) is primarily composed of a $46 \mathrm{~cm}$ diameter loop for emitting continuous sinusoidal EM fields at selected frequencies from $100 \mathrm{kHz}$ to $30 \mathrm{MHz}$. A stub dipole and a loop antenna were used to detect the electric and magnetic fields, respectively. A portable computer automatically controls the source frequency and the data recording of the electrical and the magnetic antenna signals. All units are interconnected via optical links. Pictures of the three antennae are displayed in Figure 2.

The system looks simple at first glance, however, accurate measurement of the absolute electric and magnetic field at this frequency range is extremely difficult, especially for the electric field. Our experience indicates that the electric field is vulnerable to stray pick-up caused by the wiring attached to the antenna. We have been able to identify the main part of the pick-up by repeating the measurements in reversed polarizations. The estimated pick-up is then used to make a correction to each measurement. This technique is valuable in improving data quality of the existing system, but it requires additional acquisition time for repeat measurements in reversed polarizations. Furthermore, the correction appears to be only effective in eliminating the first-order pick-up by wires. The other spurious noise caused by the interferences of protruding electronics components near the center and at the base of the receiver unit remains unchecked. To make a fundamental improvement to the existing sensor, we have redesigned it by miniaturizing the electronics components and replacing all wires with optical fibers. The redesigned and repackaged stub antenna is displayed in Figure 3. All electronic components, including power supply and amplifiers, are miniaturized, packaged together and positioned at the center of the stub antenna. All communication is done via optical fibers directly attached to the central electronics box. Figure 4 illustrates improvement in electric field measurements using the redesigned stub antenna over the old one. Figure 4(a) shows the comparison of the electric field (dots) with the old antenna in one direction and the electric field (line) with the antenna in the reversed direction. These two measurements 
are significantly different, especially at the high frequency end. However, the pick-up by the wires can be estimated by summing two measurements of opposite polarization and subtracting the residual sum from each of the individual observations to obtain the correct readings. The result is shown in Figure 4(b). The corrected data compare reasonably well with each other except at frequencies below $0.3 \mathrm{MHz}$ and around $10 \mathrm{MHz}$. Finally, the redesigned stub antenna (Figure 3) was used to measure electric fields. Figure 4(c) shows fields in reversed polarizations and they match very well for all frequencies up to 30 MHz and signal-to-noise ratio has also been improved. Notice that field characteristics in Figures 4(b) and 4(c) are different because these two sensors are of different calibration factors, which need to be investigated further. The measured magnetic field in the same polarization as the dipole source is displayed in Figure 5 and the calculated impedance using the raw electric and magnetic fields are shown in Figure 6, as well as the theoretical impedance for a two layer model at the University of California's Richmond Field Station (Frangos, 2000). Since the electric and magnetic sensors have not yet been calibrated, the impedance derived from the measured data are multiplied by 39.7 for comparison with the simulation results.

\section{Summary}

A prototype 0.1 to $30 \mathrm{MHz}$ HFI system was assembled using off-the-shelf components including a magnetic dipole transmitter, and electric and magnetic sensors. The prototype system was tested at a number of sites with different electrical properties demonstrating the proof-of-concept. Encouraged with the initial success, we started to upgrade existing electronic components and antennae so as to improve the reliability and the signal-to-noise ratio of the system. All electronic components will be made as compact as possible. Only optical links will be used to avoid picking up coherent noise from the ambient electric field. The apparent improvement in data quality can be seen from the results of the redesigned electric stub dipole. Similar upgrading is under way for the loop source and the magnetic field antenna. However, the production of power in such a compact EM source unit to obtain an appropriate dipole moment remains a difficult engineering task. To further improve the HFI system we plan to use a network analyzer for data acquisition in a swept-frequency mode so that the efficiency of the upgraded system will be greatly improved.

\section{Acknowledgements}

This work is supported by the Assistant Secretary for Environmental Restoration and Waste Management, Office of Technology Development of the U.S. Department of Energy under contract No. DE-AC03-76SF00098.

\section{References}

Frangos, W., 2000, High Frequency Impedance Measurements for Non-invasive Permittivity Determination, $\mathrm{PhD}$ Thesis, University of California, Berkeley.

Lee, K.H., and Becker, A., 2001, High frequency electromagnetic impedance measurements for characterization, monitoring and verification efforts, Interim Report, Project \#60328, U.S. DOE.

Pellerin, L., Alumbaugh, D. L., Pfeifer, M. C., 1997, The electromagnetic integrated demonstration at the INEL Cold Test Pit, in the proceedings of the Symposium on the Application of Geophysics to Engineering and Environmental Problems (SAGEEP), Reno, Nevada, March 23-26. 
Song, Y., Kim, H.J., and Lee, K.H., 2002, High-frequency electromagnetic method for subsurface imaging; Geophysics, v. 67, 501-510.

Stewart, D. C., Anderson, W. L., Grover, T. P., and Labson, V. F., 1994, Shallow subsurface mapping by electromagnetic sounding in the $300 \mathrm{kHz}$ to $30 \mathrm{MHz}$ range: Model studies and prototype system assessment: Geophysics, v. 59, 1201-1210.

Tseng, H.-W., Lee, K. H., and Becker, A., 2003, Electromagnetic impedance system for mapping the shallow subsurface: the 7th International Symposium on Recent Advances in Exploration Geophysics in Kyoto (RAEG2003), Japan.

(a)

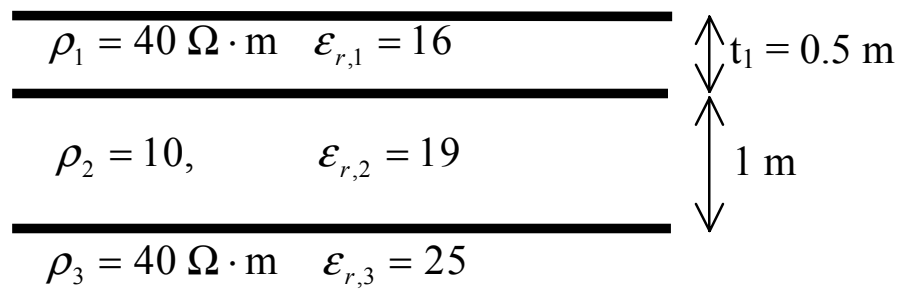

(b)

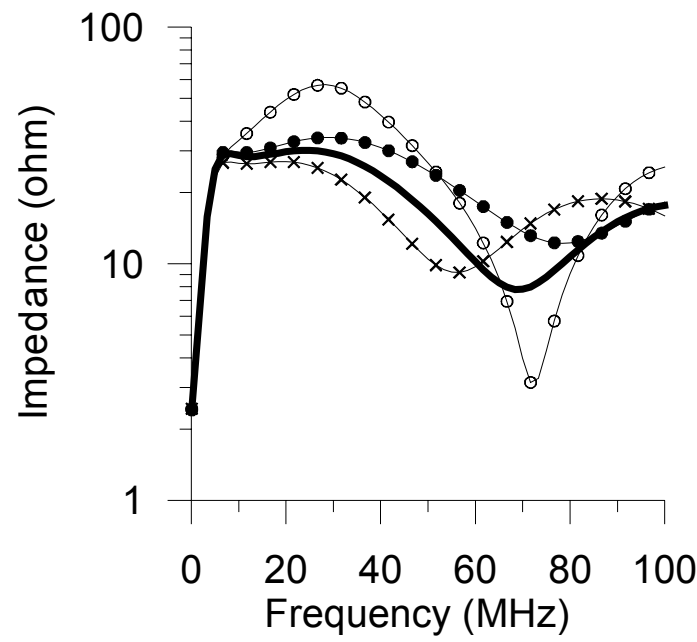

(c)

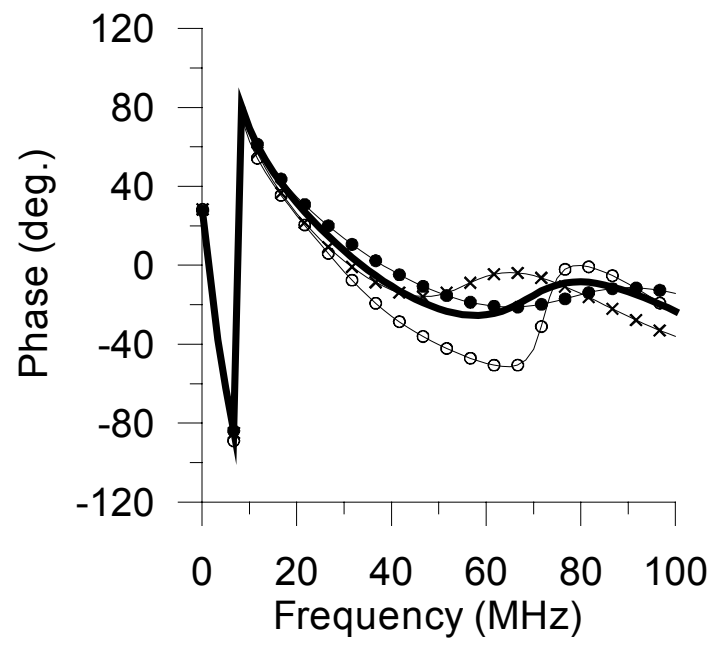

Figure 1.: (a) A 3-layered model used to simulate the near-surface electrical structure at a farm site for soil moisture monitoring; (b) and (c) Amplitude and phase of the calculated impedance, respectively. All parameters of the second and bottom layers remain fixed and only the resistivity, $\rho_{1}$, dielectric constant, $\varepsilon_{r, 1}$, and thickness of the first layer, $\mathrm{t}_{1}$, are changed. For the bold line, $\rho_{1}=40 \Omega \cdot \mathrm{m}, \varepsilon_{r, 1}=16$, $t_{1}=0.5 \mathrm{~m}$; for the solid circle, $\rho_{1}=40 \Omega \cdot \mathrm{m}, \varepsilon_{r, 1}=13, t_{1}=0.5 \mathrm{~m}$; for the open circles, $\rho_{1}=80 \Omega \cdot \mathrm{m}, \varepsilon_{r, 1}=16$, $t_{1}=0.5 \mathrm{~m}$; and for the crosses, $\rho_{1}=40 \Omega \cdot \mathrm{m}, \varepsilon_{r, 1}=13, t_{1}=0.6 \mathrm{~m}$. 
(a) Loop transmitter

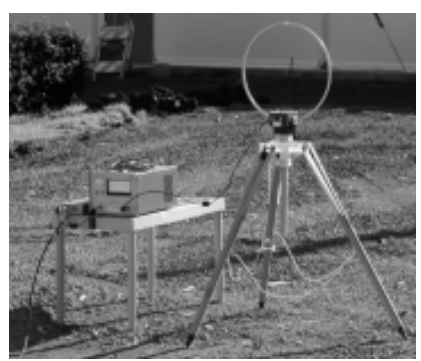

(b) Stub E-dipole

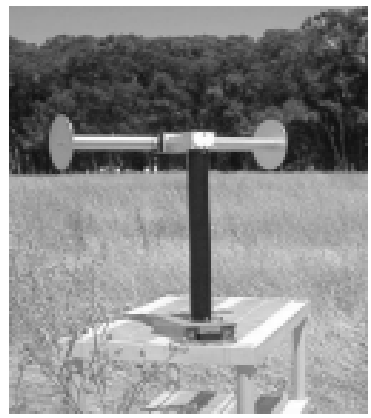

(c) Loop receiver

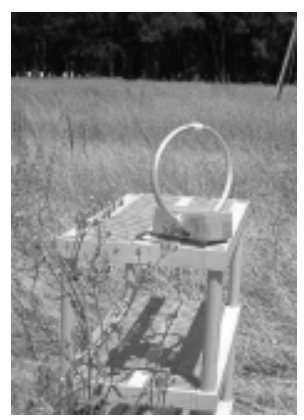

Figure 2.: HFI transmitting and receiving antennas: (a) Loop transmitter and related power supply; (b) Electric field sensor with a long supporting beam and a big base plate; (c) Magnetic field sensor.

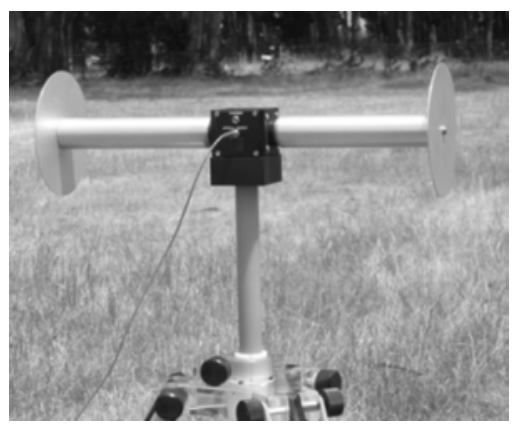

Figure 3.: Repackaged electric field sensor.

(a)

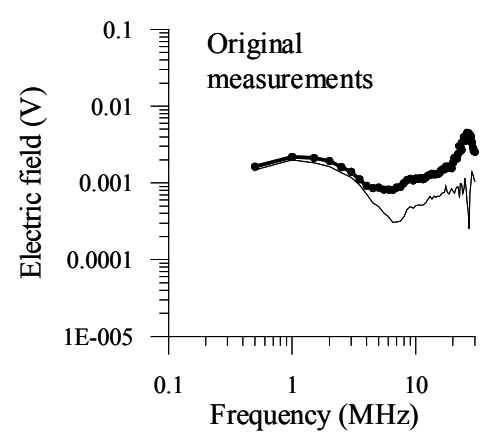

(b)

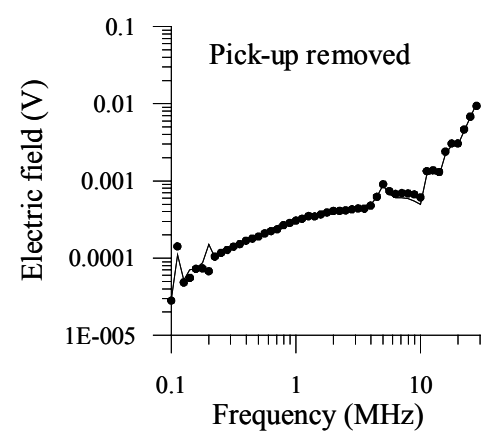

(c)

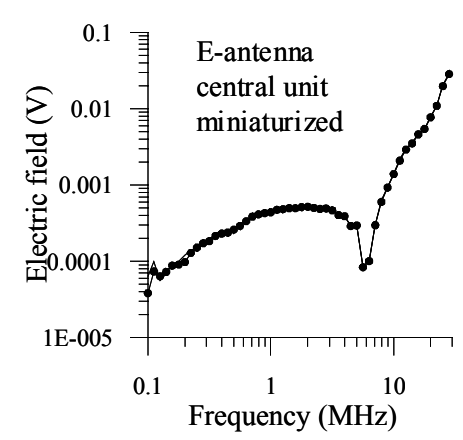

Figure 4.: A comparison of electric field measurements between the old and the miniaturized stub antennae: (a) Electric fields measured with the old antenna in normal and reversed polarization; (b) Electric fields with pick-up removed. Pick-up from stub antenna is estimated using measurements from the normal and reversed polarizations; (c) Electric fields in both normal and reversed polarization using the repackaged antenna. 


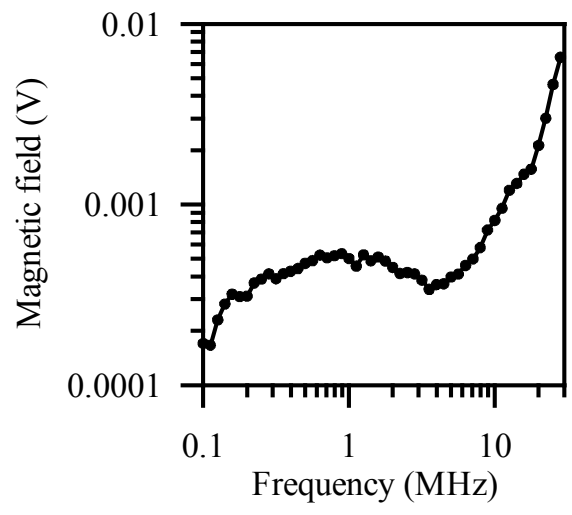

Figure 5.: Measured magnetic field, not calibrated.
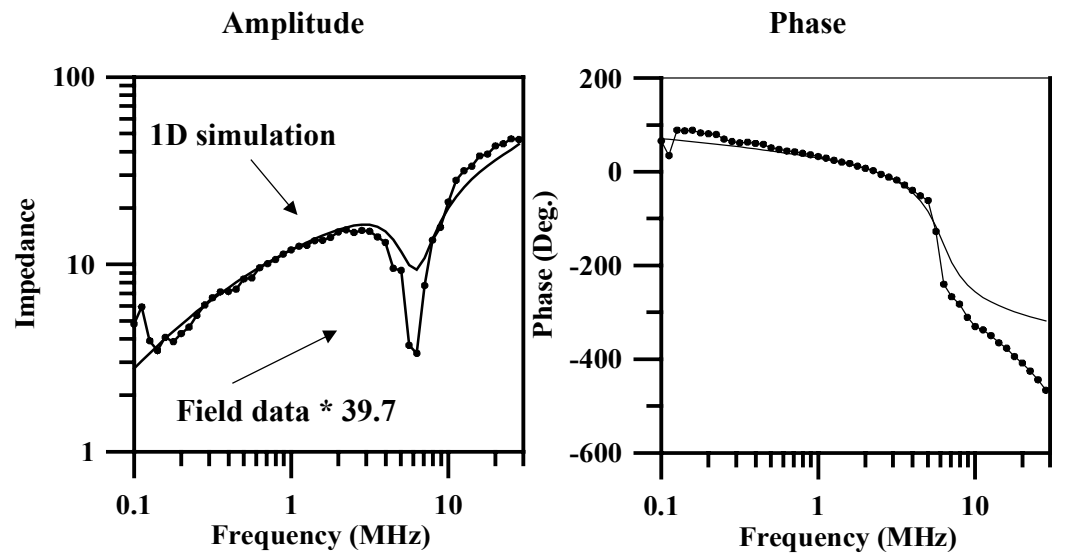

Two layer model:

$$
\begin{aligned}
& \rho=120 \Omega \cdot \mathrm{m}, \quad \varepsilon_{r}=5 \\
& \rho=45 \Omega \cdot \mathrm{m}, \quad \varepsilon_{r}=20
\end{aligned}
$$

Fig 6.: Amplitude and phase of the impedance (solid line with dots) derived from the measured electric field in Figure 4(c) and magnetic field in Figure 5. The calculated impedance for a two-layer model is indicated as a solid line. 